\title{
Mortalidade infantil em duas coortes de base populacional no Sul do Brasil: tendências e diferenciais
}

\author{
Ana M. B. Menezes 1 \\ Cesar G. Victora ${ }^{2}$ \\ Fernando C. Barros 2 \\ Elaine Albernaz 3 \\ Flávio S. Menezes 4 \\ Heitor A. Jannke 4 \\ Clarita Alves 1 \\ Cristina Rocha ${ }^{1}$
}

\author{
Infant mortality in two population-based cohorts in \\ southern Brazil: trends and differentials
}

\footnotetext{
1 Departamento de Clínica Médica, Faculdade

de Medicina, Universidade Federal de Pelotas.

C. P. 464, Pelotas, RS 96001-970, Brasil.

2 Departamento de Medicina Social, Faculdade

de Medicina, Universidade

Federal de Pelotas.

C. P. 464, Pelotas

RS 96001-970, Brasil.

3 Departamento

Materno-Infantil,

Faculdade de Medicina,

Universidade Federal

de Pelotas.

C. P. 464, Pelotas, RS

96001-970, Brasil.

${ }^{4}$ Departamento de Cirurgia

Geral, Faculdade

de Medicina, Universidade

Federal de Pelotas.

C. P. 464, Pelotas, RS 96001-970, Brasil.
}

\begin{abstract}
Time trends in infant mortality were assessed through two cohort studies carried out in Pelotas, Southern Brazil, in 1982 and 1993. Both cohorts included all hospital deliveries, and deaths were monitored through regular visits to hospitals, cemeteries, and notary publics. Information on cause of death was obtained from pediatricians, case notes, autopsies, and home visits to parents. The infant mortality rate fell from 36.4 in 1982 to 21.1 per thousand live births in 1993. The main causes of death in 1993 were perinatal, congenital malformations, diarrhea, and respiratory infections. Low birthweight babies were twelve times more likely to die than the others. The death rate among preterm babies was 2.0 times greater than for those with intrauterine growth retardation. Mortality among children whose families earned less than one minimum wage was almost seven times greater than for those with a family income of more than ten times the monthly minimum wage. Mortality for low birthweight babies from high-income families decreased by 67\%, as compared to only 36\% for those from low-income families. Thus, although mortality decreased substantially over the course of the decade, wide social differentials have persisted.
\end{abstract}

Key-words Infant Mortality; Birthweight; Family Income; Pediatric Intensive Care Unit; Epidemiology

Resumo Estudou-se a tendência temporal da mortalidade infantil através de dois estudos de coorte realizados em Pelotas, Rio Grande do Sul, em 1982 e 1993. Ambas coortes incluíram todos os nascimentos hospitalares e óbitos verificados através de visitas regulares aos hospitais, cartórios e cemitérios. As informações sobre a causa de morte foram obtidas através de entrevistas com pediatras, revisão do prontuário, necrópsias e entrevista com os pais das crianças. O coeficiente de mortalidade infantil caiu de 36,4 por mil nascidos vivos para 21,1 na década. As principais causas de mortalidade infantil em 1993 foram as perinatais, malformações congênitas, diarréia e infecções respiratórias. Crianças com baixo peso ao nascer apresentaram mortalidade 12 vezes maior do que crianças com peso adequado, e crianças pré-termo, duas vezes mais do que crianças com retardo de crescimento intra-uterino. Crianças de famílias com renda baixa (um salário mínimo) apresentaram mortalidade sete vezes superior àquelas com renda alta (10 salários mínimos). A mortalidade de crianças de baixo peso ao nascer e alta renda familiar decresceu em $67 \%$, contra apenas $36 \%$ para as de baixa renda. Conclui-se que, mesmo com uma queda expressiva da mortalidade infantil na década, persistem importantes desigualdades sociais.

Palavras-chave Mortalidade Infantil; Peso ao Nascer; Renda Familiar; Unidade de Terapia Intensiva Pediátrica; Epidemiologia 


\section{Introdução}

A mortalidade infantil pode ser determinada por múltiplas variáveis, tais como fatores sócio-econômicos e políticos, ambientais, e relativos à assistência médica, entre outros. O coeficiente de mortalidade infantil tem sido utilizado internacionalmente como um dos principais indicadores da qualidade de vida da população (Laurenti et al., 1985).

As taxas de mortalidade infantil vêm diminuindo progressivamente em muitos países em desenvolvimento. Conforme estimativas diretas obtidas do Registro Civil de nascimentos e óbitos infantis, a tendência da mortalidade infantil no Brasil na década de 1980 a 1990 foi de declínio nos primeiros dois anos, de estagnação nos dois anos seguintes e novamente de redução de 1984 para 1990 (Simões \& Monteiro, 1995). O UNICEF estima que a mortalidade infantil no Brasil tenha declinado de 70 por mil nascidos vivos em 1982 para 53 por mil em 1993 (Grant, 1985; Grant, 1995), uma queda de $24 \%$. No entanto, o Brasil foi classificado em $63^{\circ}$ lugar entre 145 países ordenados de forma decrescente em termos de mortalidade infantil, uma situação bastante constrangedora. A situação é ainda mais grave quando se discrimina a população em diferentes categorias sociais. Os enormes diferenciais entre ricos e pobres observados em nosso país têm uma repercussão direta nos coeficientes de mortalidade infantil (Victora et al., 1989).

As informações sobre os coeficientes de mortalidade infantil são provenientes do Registro Civil, de censos e pesquisas demográficas, e de estudos epidemiológicos. Embora a qualidade dos registros oficiais no Brasil venha melhorando nos últimos anos, as pesquisas epidemiológicas oferecem inúmeras vantagens para um melhor entendimento dos fatores determinantes da mortalidade infantil. Estas incluem a maior fidedignidade dos dados obtidos, a possibilidade de verificar a qualidade da informação sobre a causa de morte e de evidenciar diferenciais conforme diversos fatores de risco.

Na cidade de Pelotas, RS, foram realizados dois estudos de coorte de base populacional nos anos de 1982 e 1993 (Victora et al., 1989; Victora et al., 1996). Todas as crianças nascidas nos hospitais da cidade foram acompanhadas, havendo-se criado um sistema para detecção dos óbitos infantis. O presente artigo mostra os resultados relativos à tendência temporal da mortalidade infantil nesta década e avalia o papel da renda familiar e do peso ao nascer como determinantes da mortalidade infantil.

\section{Metodologia}

Em 1993, todos os nascimentos hospitalares ocorridos em Pelotas foram acompanhados através de um estudo longitudinal similar ao realizado em 1982, cuja metodologia foi descrita em outras publicações (Victora et al., 1989; Barros et al., 1990). As cinco maternidades da cidade foram visitadas diariamente e os 5.304 nascimentos ocorridos foram incluídos no estudo. Detalhes da metodologia constam de outros artigos (Victora et al., 1996; Victora et al., 1996).

A metodologia aqui descrita refere-se ao subestudo de mortalidade infantil. Todos os óbitos infantis foram monitorizados no período de $1^{\circ}$ de janeiro de 1993 até 31 de dezembro de 1994, quando a última criança nascida em 1993 completou um ano de idade. A mortalidade infantil incluiu os óbitos neonatais precoces (ocorridos na primeira semana de vida) e os demais ocorridos no primeiro ano (neonatais tardios - de sete a 27 dias de idade - e pós-neonatais - até 364 dias de vida).

Os óbitos foram monitorizados diariamente nos principais hospitais da cidade, onde eram visitadas as três Unidades de Tratamento Intensivo nas quais ocorria a maioria dos falecimentos. Os berçários, enfermarias pediátricas e serviços de Pronto Socorro dos demais hospitais eram visitados quinzenalmente, pois raramente ocorriam óbitos nesses locais. Com o intuito de detectar óbitos extra-hospitalares, mensalmente eram visitados os cartórios, cemitérios e a Delegacia Regional de Saúde.

Informações sobre a causa básica do óbito eram coletadas através de uma entrevista com o pediatra responsável pelo caso e, no caso de crianças hospitalizadas, por meio de uma revisão sistemática do prontuário hospitalar, sendo colhidas informações sobre motivo da internação, história e evolução da doença, exames complementares, tratamento e diagnóstico. Se necessário, as informações obtidas pelo questionário perinatal aplicado à mãe, logo após o nascimento, eram também utilizadas.

Para crianças falecidas entre os sete e os 364 dias de vida, realizava-se uma entrevista médica domiciliar com a mãe, quando era investigada a história clínica da doença e seus antecedentes. Essa investigação era baseada em questionários utilizados na Investigação Inter-Americana de Mortalidade na Infância (Puffer \& Serrano, 1973), adaptados para o presente estudo.

Tentou-se obter necrópsias para todas as mortes hospitalares. As necrópsias eram realizadas somente com o consentimento por es- 
crito do responsável pela criança e solicitadas pelo pediatra que havia atendido ao óbito. $\mathrm{O}$ Laboratório de Patologia da Faculdade de Medicina era responsável pela execução das necrópsias diariamente, inclusive nos fins de semana. A funerária da Prefeitura Municipal de Pelotas fazia o transporte gratuito do corpo até o Laboratório de Patologia.

Além do atestado de óbito oficial, preenchido pelo médico que assistiu a criança, dois árbitros da equipe da pesquisa revisavam todas as informações supracitadas e preenchiam independentemente dois outros atestados. Havendo discordância entre os mesmos, um terceiro árbitro era responsável pelo diagnóstico final. Os atestados eram codificados conforme a Nona Versão da Classificação Internacional de Doenças pelo coordenador da Divisão de Informação em Saúde da Secretaria da Saúde e do Meio Ambiente do Rio Grande do Sul (OMS, 1980). Os seguintes grupos de causas de óbito foram utilizados na análise: causas perinatais (códigos CID 760-779.9), malformações congênitas (códigos CID 740-759.8), infecções respiratórias (códigos CID 460-519.9), gastroenterites (códigos CID 001-009.3), outras infecções (códigos CID 090), causas mal definidas (códigos CID 780-799.9), outras causas.

Dois óbitos pós-neonatais não foram incluídos na análise. O primeiro por ter a mãe recusado a entrevista perinatal no início do estudo, e o segundo por ter sido impossível localizar o endereço (a detecção deste óbito foi através da revisão mensal na Delegacia Regional de Saúde).

O coeficiente de mortalidade infantil com os seus dois componentes, neonatal e pós-neonatal, foram as variáveis dependentes do estudo, sendo comparadas aos coeficientes encontrados em 1982. Nas publicações originais de 1982, as taxas de mortalidade foram expressas em termos de crianças-ano em risco, e não de nascidos vivos. As taxas de 1982 foram, portanto, recalculadas para permitir a comparação com 1993, razão pela qual diferem ligeiramente (em cerca de $7 \%$ ) daquelas apresentadas em publicações anteriores.

As variáveis independentes utilizadas foram o sexo, a renda familiar expressa em salários mínimos mensais, o peso ao nascer em gramas e a idade gestacional aferida através da data da última menstruação. A descrição detalhada da coleta destas variáveis e os números de crianças em cada categoria encontram-se em outro artigo no presente volume (Victora et al., 1996). Para testar associação entre as variáveis independentes e os coeficientes de mortalidade foi utilizado o teste do Qui-Quadrado.

\section{Resultados}

Ocorreram 111 óbitos infantis em Pelotas em 1993, contra 215 em 1982 (Tabela 1). O coeficiente de mortalidade infantil caiu de 36,4 para 21,1 por mil nascidos vivos, uma redução de $42 \%$, principalmente às custas do componente de óbitos pós-neonatais, cuja queda foi de $57 \%$.

Dos 111 óbitos infantis, 21 aconteceram no domicílio, sendo um neonatal precoce, cinco neonatais tardios e 15 pós-neonatais. Quatro óbitos neonatais precoces aconteceram nos berçários; um óbito neonatal tardio e dois óbitos pós-neonatais, nas enfermarias pediátricas. Os demais óbitos ocorreram nas UTIs.

Tabela 1

Mortalidade infantil em Pelotas, 1982-1993.

\begin{tabular}{lcc}
\hline Indicadores & 1982 & 1993 \\
& $\mathrm{n}$ & $\mathrm{n}$ \\
\hline Número de nascidos vivos & 5.914 & 5.249 \\
& & \\
óbitos neonatais & 119 & 75 \\
$\quad$ Número & 20,1 & 14,3 \\
Coeficientes por mil & $(55 \%)$ & $(68 \%)$ \\
\% dos óbitos infantis & & \\
& 96 & 36 \\
óbitos pós-neonatais & 16,2 & 6,9 \\
Número & $(45 \%)$ & $(32 \%)$ \\
Coeficientes por mil & & \\
\% dos óbitos infantis & 215 & 111 \\
óbitos infantis & 36,4 & 21,1 \\
Número & $(100 \%)$ & $(100 \%)$ \\
Coeficientes por mil & & \\
\% dos óbitos infantis & &
\end{tabular}

Foi possível realizar necrópsias em apenas $18 \%$ dos óbitos infantis, pois houve recusa por parte dos pais de todas as crianças falecidas no período neonatal tardio e pós-neonatal. O subregistro para mortalidade infantil foi de apenas 5,4\% comparado com $24 \%$ em 1982.

Quanto às causas de mortalidade infantil (Tabela 2), as reduções entre 1982 e 1993 foram de $28 \%$ para as causas perinatais, $60 \%$ para diarréia, 69\% para infecções respiratórias, 93\% para outras infecções e $73 \%$ para causas mal definidas. Malformações congênitas e as demais causas praticamente não sofreram alterações nesse período. As causas perinatais e as malformações, agrupadas, representaram 55\% dos óbitos em 1982 e $74 \%$ em 1993.

A redução do coeficiente de mortalidade infantil para os meninos (Tabela 3) foi de $34 \%$ contra $49 \%$ para as meninas; esta diferença 
Número de óbitos para mortalidade infantil específicos por causa em Pelotas, 1982-1993.

\begin{tabular}{lcccccc}
\hline Causas & \multicolumn{7}{c}{ Mortalidade Infantil } & & \\
& 1982 & 1993 & 1982 & 1993 & $\begin{array}{c}1982 \\
\text { Coefic. }\end{array}$ \\
& $n$ & $n$ & $\%$ & $\%$ & $\begin{array}{c}1993 \\
\text { por mil } \\
\text { por mil }\end{array}$ \\
& & & & & & \\
\hline Perinatais & 92 & 57 & 43 & 51 & 15,4 & 11,1 \\
Malformações & 27 & 25 & 13 & 23 & 4,5 & 4,8 \\
Diarréia & 25 & 9 & 12 & 8 & 4,2 & 1,7 \\
Infecções respiratórias & 25 & 7 & 12 & 6 & 4,2 & 1,3 \\
Outras infecções & 18 & 1 & 8 & 1 & 3,0 & 0,2 \\
Outras causas & 4 & 5 & 2 & 5 & 0,7 & 1,0 \\
Mal definidas & 24 & 7 & 11 & 6 & 4,0 & 1,1 \\
Total & 215 & 111 & 100 & 100 & 36,0 & 21,1 \\
\hline
\end{tabular}

Coefic.: coeficiente

Tabela 3

Coeficientes de mortalidade infantil (por mil) conforme sexo em Pelotas, 1982-1993.

\begin{tabular}{|c|c|c|c|c|c|c|c|c|}
\hline \multirow[t]{2}{*}{ Variável } & \multicolumn{2}{|c|}{ Neonatal } & \multicolumn{2}{|c|}{ Pós-neonatal } & \multicolumn{2}{|c|}{ Infantil } & \multicolumn{2}{|c|}{ n nascimentos } \\
\hline & 1982 & 1993 & 1982 & 1993 & 1982 & 1993 & 1982 & 1993 \\
\hline \multicolumn{9}{|l|}{ Sexo } \\
\hline Masculino & 22,1 & 17,8 & 15,8 & 7,4 & 38,2 & 25,2 & 3.083 & 2.603 \\
\hline Feminino & 17,4 & 10,5 & 16,0 & 6,4 & 33,3 & 16,9 & 2.926 & 2.687 \\
\hline$p^{*}$ & $>0,05$ & $<0,05$ & $>0,05$ & $>0,05$ & $>0,05$ & $<0,05$ & & \\
\hline
\end{tabular}

* teste $\chi^{2}$

Tabela 4

Coeficientes de mortalidade infantil (por mil) conforme o peso ao nascer e a idade gestacional (data da última menstruação) em Pelotas, 1982-1993.

\begin{tabular}{|c|c|c|c|c|c|c|c|c|}
\hline \multirow{3}{*}{$\begin{array}{l}\text { Peso ao nascer e } \\
\text { idade gestacional }\end{array}$} & \multicolumn{8}{|c|}{ Coeficientes de mortalidade } \\
\hline & \multicolumn{2}{|c|}{ Neonatal } & \multicolumn{2}{|c|}{ Pós-neonatal } & \multicolumn{2}{|c|}{ Infantil } & \multicolumn{2}{|c|}{ Óbitos/Nascim. } \\
\hline & 1982 & 1993 & 1982 & 1993 & 1982 & 1993 & 1982 & 1993 \\
\hline $\begin{array}{l}\text { Pequenas para } \\
\text { a idade gestacional }\end{array}$ & 38 & 14 & 29 & 12 & 66 & 26 & $40 / 693$ & $20 / 779$ \\
\hline \multicolumn{9}{|l|}{$\begin{array}{l}\text { Peso adequado para } \\
\text { a idade gestacional: }\end{array}$} \\
\hline pré-termo & 97 & 42 & 65 & 16 & 162 & 57 & $37 / 255$ & $29 / 505$ \\
\hline a termo & 6 & 2 & 8 & 3 & 15 & 5 & $49 / 3.718$ & $17 / 3.375$ \\
\hline$p^{*}$ & $<0,001$ & $<0,001$ & $<0,001$ & 0,001 & $<0,001$ & $<0,001$ & & \\
\hline
\end{tabular}

* teste $\chi^{2}$ 
aconteceu no período neonatal, pois no período pós-neonatal ambos os sexos tiveram redução semelhante.

A Figura 1 mostra a mortalidade neonatal e pós-neonatal segundo o peso ao nascer, em 1982 e 1993. Em 1982, crianças de baixo peso ao nascer $(<2.500 \mathrm{~g})$ apresentavam um risco 25 vezes maior para a mortalidade neonatal do que crianças nascidas com peso adequado ( $\geq 2.500$ g), sendo que, em 1993, este risco relativo foi de 23 vezes. Quanto ao componente pós-neonatal, em 1982 o risco relativo foi de 4,5 para crianças de baixo peso ao nascer, ao passo que, em 1993, este foi de três vezes.

Em 1982, o coeficiente de mortalidade infantil, dado pela soma dos componentes neonatal e pós-neonatal, foi de 206 por mil para as crianças de baixo peso e de 18 por mil para as de peso adequado. Os respectivos coeficientes para 1993 foram 114 e 10 por mil.

Crianças com baixo peso ao nascer incluem aquelas com retardo de crescimento intra-uterino e aquelas nascidas com menos de $37 \mathrm{se}$ manas de gestação. Tanto em 1982 como em 1993, a mortalidade das crianças pré-termo foi cerca de 2,5 vezes maior do que a das crianças com retardo de crescimento (Tabela 4), embora o peso médio das crianças pré-termo tenha sido superior ao dos últimos. Uma exceção foi a mortalidade pós-neonatal em 1993, cujo risco relativo foi de 1,3 (16/12). Comparando 1982 com 1993, a queda na mortalidade de todos os grupos foi de cerca de três vezes, com exceção novamente da mortalidade pós-neonatal em crianças pré-termo, que caiu cerca de quatro vezes. Crianças sem retardo, nascidas a termo, apresentaram índices muito baixos de mortalidade. Estes resultados devem ser interpretados com cautela devido ao número relativamente pequeno de óbitos, particularmente os pósneonatais em 1993.

A mortalidade infantil, conforme cinco categorias de renda familiar, é mostrada na Figura 2. Para todos os grupos de renda, a mortalidade em 1993 foi inferior à de 1982. Crianças de famílias de baixa renda (um salário mínimo), tanto em 1982 quanto em 1993, apresentaram risco seis vezes mais alto do que crianças de famílias de alta renda (> 10 salários mínimos). Portanto, apesar de uma redução geral nos níveis de mortalidade, as desigualdades sociais permaneceram.

A mortalidade infantil foi estudada para as diferentes combinações de renda familiar (expressa em duas categorias: três ou acima de três salários mínimos) e peso ao nascer (Figura 3). Em 1982 houve uma interação entre estas duas variáveis: entre crianças de baixo peso, a
Figura 1

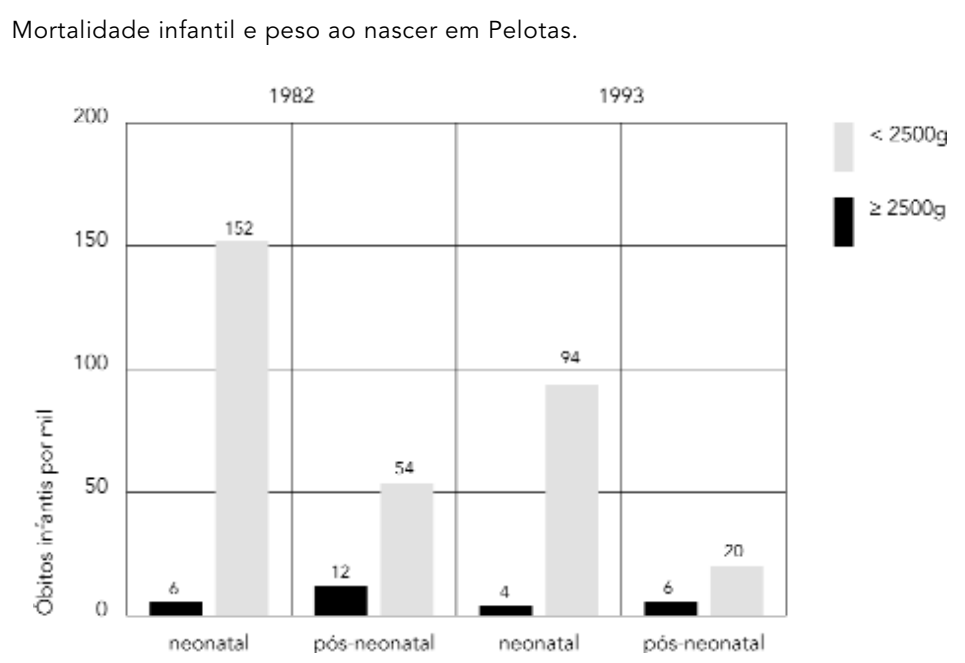

Figura 2

Mortalidade infantil conforme a renda familiar em Pelotas.

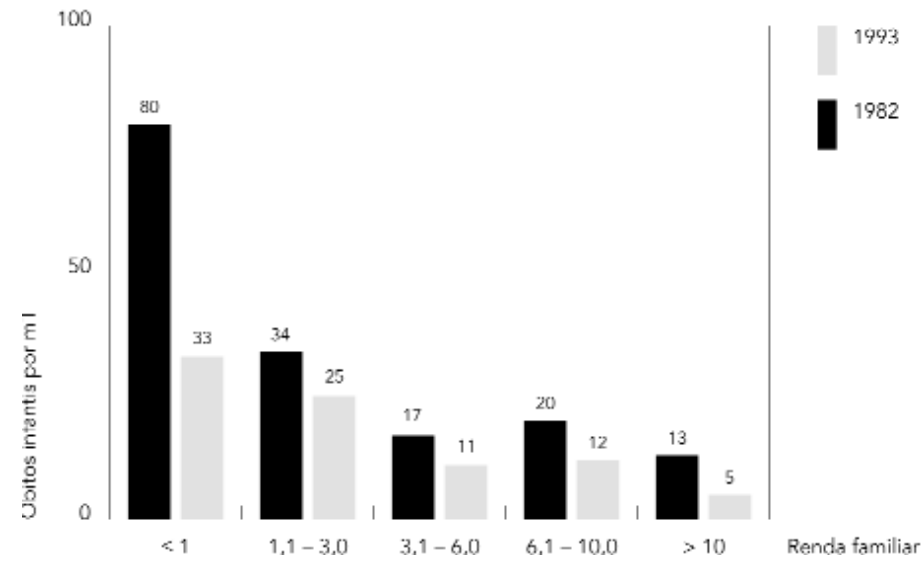

Figura 3

Mortalidade infantil conforme a renda e peso em Pelotas.

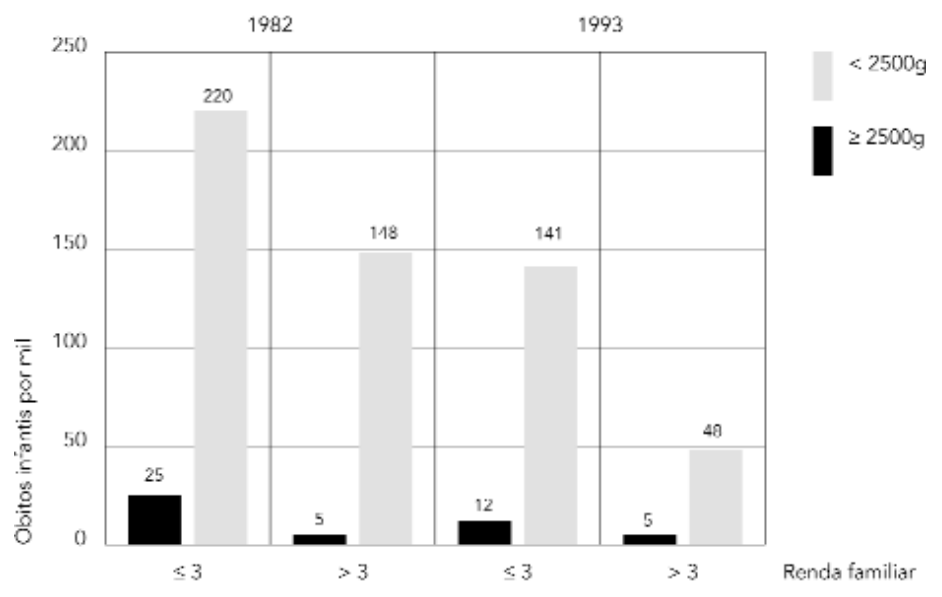


mortalidade para o grupo de baixa renda foi 1,5 maior do que para as de alta renda. Para crianças de peso adequado, este risco relativo foi igual a 5,0. Já em 1993, a situação foi bastante diferente: a mortalidade do grupo de alta renda foi cerca de três vezes menor do que a das demais crianças, independente do peso ao nascer.

Outra forma de examinar estes dados é comparar as tendências entre 1982 e 1993 para cada combinação de peso e renda. Crianças com baixo peso de famílias de baixa renda mostraram uma redução de $36 \%$ da mortalidade infantil de 1982 para 1993; aquelas nascidas com baixo peso, mas pertencentes a famílias de alta renda tiveram $67 \%$ de redução. Para crianças nascidas com peso adequado de famílias de baixa renda, o coeficiente de mortalidade diminuiu de 25 para 12; para crianças de famílias de alta renda, o coeficente já em 1982 havia atingido níveis extremamente baixos, permanecendo quase inalterado na década.

\section{Discussão}

A existência de dois estudos de coorte com base populacional, realizados com um intervalo de cerca de uma década, apresenta uma oportunidade única para estudar tendências e diferenciais de mortalidade. Ambos os estudos incluíram praticamente todos os nascimentos hospitalares ocorridos na cidade no período de um ano. Partos domiciliares são extremamente raros, representando menos de $1 \%$ do total, o que garante a base populacional do estudo hospitalar.

Para a avaliação dos coeficientes de mortalidade como indicadores de saúde é preciso um adequado registro dos óbitos, o que não ocorre em muitos países em desenvolvimento. No presente estudo, os óbitos foram detectados através de múltiplas fontes, sendo, portanto, pouco provável que a equipe deixasse de identificar alguma criança falecida. Encontrou-se, em 1993, um sub-registro para mortalidade infantil de apenas 5,4\%, comparado ao alto nível de $24 \%$ detectado em 1982 (Barros et al., 1985). A marcada redução no nível de sub-registro pode ter sido conseqüência, pelo menos parcialmente, do próprio estudo de 1982, pois, devido aos achados daquele estudo, foi realizada uma série de reuniões entre os pesquisadores, as autoridades locais de saúde, os titulares dos Cartórios de Registro Civil e os encarregados dos cemitérios da cidade. Descobriu-se que muitas crianças eram enterradas com a sim- ples apresentação da Declaração de óbito fornecida pelo médico, sem que houvesse o registro no cartório. Os administradores dos cemitérios passaram, então, a exigir este último documento, o que reduziu substancialmente o sub-registro.

No presente estudo, houve um intenso esforço para se obter necrópsias das crianças falecidas, sendo organizada uma estrutura que iniciava com a notificação do óbito à coordenadora do estudo, passava pela solicitação da necrópsia pelo pediatra, pela autorização familiar, pelo transporte gratuito do corpo e incluía a disponibilidade permanente de patologistas. Apesar de todo este esforço organizacional, a resistência dos familiares foi marcada, sendo possível autopsiar apenas $18 \%$ dos óbitos infantis. Este resultado sugere que, infelizmente, estudos baseados em necrópsias continuarão sendo raros em nosso meio.

Houve uma importante redução do coeficiente de mortalidade infantil em Pelotas $(42 \%)$ na última década, principalmente às custas dos óbitos pós-neonatais (redução de $57 \%)$. Uma queda com igual magnitude (42\%) foi observada, também, no Estado do Rio Grande do Sul (DIS/ATMA/RS, 1995), sendo a redução para o país como um todo de $24 \%$ segundo o UNICEF (Grant, 1985; Grant, 1995). As possíveis razões para a queda da mortalidade observada em Pelotas são discutidas no último artigo desse suplemento (Barros et al., 1996).

Apesar destas importantes quedas, observa-se ainda um alto coeficiente de mortalidade infantil no país. O UNICEF estima, com base em diferentes fontes, incluindo métodos indiretos, que a mortalidade infantil no Brasil tenha sido igual a 53 por mil em 1993 (Grant, 1995). Para o Rio Grande do Sul (DIS/ATMA/RS, 1995) e Pelotas, os coeficientes foram respectivamente 19,2 e 21,1 por mil, níveis ainda quatro vezes superiores aos observados nos países de mais baixa mortalidade infantil - os países escandinavos e países industrializados do Extremo Oriente -, onde apenas cinco a seis em cada mil crianças morrem no primeiro ano (Grant, 1995).

Tradicionalmente, a queda na mortalidade infantil se dá principalmente às custas do componente pós-neonatal (Pharoah \& Morris, 1979), provavelmente pela diminuição das infecções, especialmente a diarréia e a infecção respiratória. Para alguns países da América Latina, no entanto, após alguns anos de queda do componente pós-neonatal, este alcançou níveis bastante baixos, a partir dos quais reduções adicionais são difíceis. A mortalidade pós- 
neonatal tende então a se estabilizar, enquanto o componente neonatal passa a cair mais rapidamente (Guzmán \& Orellana, 1989). Pelotas parece não haver atingido ainda este último estágio.

Nos países com as menores taxas de mortalidade infantil, as malformações congênitas ocupam o primeiro lugar como causa de mortalidade infantil. Mesmo no período pós-neonatal, as malformações são responsáveis por metade das mortes (Pharoah \& Morris, 1979). As maiores taxas de redução em Pelotas atingiram as doenças infecciosas: infecções respiratórias, diarréia e outras infecções. Redução importante $(73 \%)$ também ocorreu para as causas mal definidas, o que provavelmente deveu-se ao cuidado na averiguação da causa de óbito. As causas perinatais e as malformações passaram assim a ser responsáveis por três quartos dos óbitos em 1993. Estas causas são de mais difícil prevenção do que as restantes, e portanto assumem um percentual mais alto com a queda dos índices de mortalidade.

Tanto em 1982 como em 1993, observou-se que o baixo nível sócio-econômico e baixo peso ao nascer estiveram fortemente associados à mortalidade infantil. Embora a mortalidade tenha caído em todos os grupos de renda, os níveis seguem sendo cerca de seis vezes maiores entre as crianças mais pobres do que entre as mais ricas. Estas últimas atingiram, em 1993, o nível de cinco óbitos por mil nascidos vivos, similar ao observado nos países de mais baixa mortalidade (Grant, 1995). Embora a existência de importantes diferenciais sócio-econômicos na mortalidade infantil tenha sido amplamente documentada, é necessário continuar a denunciar tais diferenciais enquanto persistirem.

$\mathrm{O}$ peso ao nascer continua sendo o mais importante fator de risco diretamente ligado à mortalidade infantil. Crianças de baixo peso seguem morrendo 12 vezes mais do que as de peso adequado. Entre as crianças de baixo peso, as nascidas pré-termo apresentam risco substancialmente maior do que as com retardo de crescimento intra-uterino. Isto comprova a necessidade de intervenções pré-natais para prevenir os partos pré-termo, como o controle de infecções (Ferraz et al., 1990).

Os presentes resultados não evidenciam um claro impacto das unidades de tratamento intensivo (UTIs) pediátricas sobre a mortalidade infantil. Em 1982, não existiam UTIs pediátricas na cidade, sendo três unidades criadas durante a década nas maiores maternidades. A mortalidade neonatal entre as crianças de baixo peso caiu de 152 para 94 por mil, achado que sugere um possível impacto das UTIs. No entanto, a mortalidade entre crianças de peso adequado também caiu de seis para quatro por mil, embora os números de óbitos tenham sido pequenos (34 e 17, respectivamente). Dois outros argumentos questionam a efetividade das UTIs. A maior queda na mortalidade infantil deu-se às custas do componente pós-neonatal, e não do neonatal, no qual esperar-se-ia um maior impacto. Em segundo lugar, a queda na mortalidade neonatal foi de igual magnitude cerca de 2,5 a três vezes - para crianças com ou sem retardo de crescimento intra-uterino e para as nascidas pré-termo, ao passo que seria lícito esperar uma maior redução entre estas últimas. Portanto, não se observa um impacto claro com a introdução das UTIs.

O estudo da mortalidade infantil estratificada simultaneamente por renda familiar, peso ao nascer e ano do estudo (Figura 3 ) foi bastante revelador. O principal achado foi a diferença na queda de mortalidade entre crianças com baixo peso ao nascer. Entre famílias de baixa renda, a redução foi de $36 \%$, sendo esta de $67 \%$ para o grupo de alta renda. Se a queda na mortalidade for atribuída a melhores cuidados médicos, incluindo o uso das UTIs, estes achados sugerem que o grupo de alta renda tem sido mais beneficiado do que o das crianças pobres. Discussões informais com pediatras envolvidos em cuidados intensivos neonatais sugerem ser o atendimento dentro destas unidades de fato diferenciado, em termos tanto de atendimento de médico particular e de utilização de equipamentos mais sofisticados, quanto da própria presença da mãe dentro da unidade.

Os presentes achados apontam para uma expressiva queda da mortalidade infantil em Pelotas na década, o que também vem sendo evidenciado no Rio Grande do Sul e, em menor intensidade, em todo o país. Entretanto, as diferenças sociais existentes em 1982 permanecem com a mesma magnitude em 1993. Isso mostra ser ainda possível reduzir a mortalidade infantil através da diminuição das desigualdades sociais e de cuidados médicos adequados para todas as crianças em risco. 


\section{Referências}

BARROS, F. C.; VICTORA, C. G.; TEIXEIRA, A. M. B. \& PUERTO FILHO, M., 1985. Mortalidade perinatal e infantil em Pelotas, Rio Grande do Sul: nossas estatísticas são confiáveis? Cadernos de Saúde Pública, 1:348-358.

BARROS, F. C.; VICTORA, C. G. \& VAUGHAN, J. P., 1990. The Pelotas birth cohort study, 1982-1987. Strategies for following up 6,000 children in a developing country. Perinatal Pediatric Epidemiology, 4:267-1282.

BARROS, F. C.; VICTORA, C. G.; TOMASI, E.; HORTA, B. L.; MENEZES, A. M.; CESAR, J. A; HALPERN, R.; OLINTO, M. T.; POST, C. L.; COSTA, J. S. D.; MENEZES, F; GARCIA, M. Del M. \& VAUGHAN, J. P., 1996. Tendências e diferenciais na saúde materno-infantil em Pelotas, RS: conclusões e recomendações. Cadernos de Saúde Pública, 12(supl. 1):714.

DIS/ATMA/RS (Divisão de Informação em Saúde/Assessoria Técnica e do Meio Ambiente/Rio Grande do Sul), 1995. Estatísticas de Saúde: Mortalidade. Porto Alegre: Secretaria da Saúde e Meio Ambiente.

FERRAZ, E. M.; GRAY, R. H. \& CUNHA, T. M., 1990 Determinants of preterm delivery and intrauterine growth retardation in North-East Brazil. International Journal of Epidemiology, 19:101-107.

GRANT, J., 1985. Situação Mundial da Infância 1985. Brasília: UNICEF.

GRANT, J., 1995. Situação Mundial da Infância 1995. Brasília: UNICEF.

GUZMÁN, J. M. \& ORELLANA, M., 1989. Nuevas tendencias de mortalidad infantil en Cuba, Chile y Costa Rica. In: Salud, Enfermedad y Muerte de los Niños en America Latina. (Clacso/IDRC, eds.), pp. 343-378, Otawa: Consejo Latinoamericano de Ciencias Sociales (Clacso) e International Development Research Centre (IDRC).

LAURENTI, R.; JORGE, M. H. P.; LEBRÃO, M. L. \& GOTLIEB, S. L. D., 1985. Estatísticas de Saúde. São Paulo: EPU/EDUSP.
OMS (Organização Mundial de Saúde), 1980. Classificação Internacional de Doenças. Revisão 1975. São Paulo: Centro da OMS para Classificação de Doenças em Português.

PHAROAH, P. O. D. \& MORRIS, J. N., 1979. Postneonatal mortality. Epidemiologic Reviews, 1:170-183.

PUFFER, R. R. \& SERRANO, C.V., 1973. Patterns of Mortality in Childhood: The Inter-American Investigation of Mortality in Childhood. Scientific Publication no 262. Washington: PAHO.

SIMÕES, C. C. S. \& MONTEIRO, C. A., 1995. Tendência secular e diferenciais regionais da mortalidade infantil no Brasil. In: Velhos e Novos Males da Saúde no Brasil. A Evolução do País e de Suas Doenças (C. A. Monteiro, org.), pp. 151-156, São Paulo: Hucitec.

VICTORA, C. G.; BARROS, F. C. \& VAUGHAN, J. P. 1989. Epidemiologia da Desigualdade: Um Estudo Longitudinal de 6.000 Crianças Brasileiras. 2a ed., São Paulo: CEBES/Hucitec.

VICTORA, C. G.; BARROS, F. C.; HALPERN, R.; MENEZES, A. M.; HORTA, B. L.; TOMASI, E.; WEIDERPASS, E.; CESAR, J. A.; OLINTO, M. T.; GUIMARÃES, P. R. V.; GARCIA, M. Del M. \& VAUGHAN, J. P., 1996. Estudo longitudinal da população materno-infantil de Pelotas, RS, 1993: aspectos metodológicos e resultados preliminares. Revista de Saúde Pública, 30:34-35.

VICTORA, C. G.; BARROS, F. C.; TOMASI, E.; MENEZES, A. M.; HORTA, B. L.; WEIDERPASS, E.; CESAR, A. J.; COSTA, J. S. D.; OLINTO, M. T.; HALPERN, R.; GARCIA, M. Del M. \& VAUGHAN, J. P., 1996. Tendências e diferenciais na saúde materno-infantil: delineamento e metodologia das coortes de 1982 e 1993 de mães e crianças de Pelotas, RS. Cadernos de Saúde Pública, 12(supl. 1):7-14 